\title{
Perinatal Transmission of HIV in Women Receiving Antiretroviral Prophylaxis/Therapy during Pregnancy with Zidovudine, Lamivudine, and Nevirapine: Experience at Tertiary Care Center in India
}

\author{
${ }^{1}$ Khushbu Meena, ${ }^{2}$ Aashima Arora, ${ }^{3}$ Rashmi Bagga, ${ }^{4}$ Jasvinder Kalra, ${ }^{5}$ Praveen Kumar, ${ }^{6}$ Aman Sharma, ${ }^{7}$ Sunil Arora
}

\begin{abstract}
Objective: To compare the perinatal transmission rate, maternal and neonatal outcome among HIV positive women on triple drug antiretroviral therapy (ART) with women receiving antiretroviral (ARV) prophylaxis for prevention of mother to child transmission (MTCT).
\end{abstract}

Materials and methods: This prospective study recruited all HIV positive antenatal women who delivered at Postgraduate Institute of Medical Education and Research, Chandigarh from June 2010 to December 2011. The rate of HIV perinatal transmission was calculated in the two groups. The perinatal outcome was studied in the form of birth weight, gestation at delivery, mode of delivery, maternal morbidity and mortality.

Results: The overall HIV transmission rate was $13.3 \%$ (10\% in ART group and $15 \%$ in ARV group). A trend was seen toward a lower MTCT among women in ARV prophylaxis group who delivered by elective cesarean section (CS) as compared to vaginal delivery (7.7 vs $16.7 \%$ ).

Conclusion: The vertical transmission rate of HIV was lower among women receiving triple drug ART. The mode of delivery did not affect the transmission rate in this group.

Keywords: ART, HIV, Perinatal transmission.

How to cite this article: Meena K, Arora A, Bagga R, Kalra J, Kumar P, Sharma A, Arora S. Perinatal Transmission of HIV in Women Receiving Antiretroviral Prophylaxis/Therapy during Pregnancy with Zidovudine, Lamivudine, and Nevirapine: Experience at Tertiary Care Center in India. J Postgrad Med Edu Res 2015;49(3):115-120.

\footnotetext{
${ }^{1}$ Senior Resident, ${ }^{2}$ Research Officer, ${ }^{3-5,7}$ Professor

${ }^{6}$ Assistant Professor

${ }^{1-4}$ Department of Obstetrics and Gynecology, Postgraduate Institute of Medical Education and Research, Chandigarh India

${ }^{5}$ Department of Pediatrics, Postgraduate Institute of Medical Education and Research, Chandigarh, India

${ }^{6}$ Department of Internal Medicine, Postgraduate Institute of Medical Education and Research, Chandigarh, India

${ }^{7}$ Department of Immunopathology, Postgraduate Institute of Medical Education and Research, Chandigarh, India

Corresponding Author: Aashima Arora, Research Officer Department of Obstetrics and Gynecology, Postgraduate Institute of Medical Education and Research, Chandigarh India, Phone: 01722756345, e-mail: aashicool84@gmail.com
}

Source of support: Nil

Conflict of interest: None

\section{INTRODUCTION}

There are approximately 27 million pregnancies per year in India. With an antenatal HIV prevalence of $0.7 \%$, nearly $1,89,000$ HIV positive women deliver each year. As the vertical transmission rate is around $30 \%$, about 56,700 HIV infected infants are born every year. ${ }^{1}$ Mother to child transmission (MTCT) can occur during pregnancy, labor $(5-10 \%)$, delivery $(10-15 \%)$, and breastfeeding (5-20\%). Mother to child transmission may be reduced but not totally eliminated by antiretroviral (ARV) prophylaxis during pregnancy, labor, and to the neonate, delivery by scheduled cesarean section (CS) before labor onset or ruptured membranes and avoiding breastfeeding. ${ }^{2}$

Multiple regimens of antiretroviral drugs are available for use in pregnancy to reduce vertical transmission. Out of the numerous World Health Organization (WHO) recommended abbreviated regimens of ARV prophylaxis to be used in resource-limited countries, the national AIDS control organization (NACO), India, had selected the single-dose Nevirapine (sdNVP) regimen. ${ }^{1}$ This was provided free of cost at all integrated counseling and testing centers (ICTCs). In 2006, the WHO recommended a more effective abbreviated ARV prophylaxis regimen. ${ }^{3}$ In 2013, NACO released fresh guidelines uniformly prescribing triple drug ART to all pregnant women with HIV infection. ${ }^{4}$ Scheduled delivery by CS is recommended in women with HIV RNA levels $>1,000$ copies $/ \mathrm{ml}$ or unknown levels whether or not receiving ARVs. ${ }^{5}$ However, NACO recommends CS only for obstetric indications and not for sole prevention of MTCT in view of the morbidity and expense of a CS and limited availability of safe blood transfusion. ${ }^{1}$ World Health Organization recommends exclusive breastfeeding to the newborn for first 6 months unless replacement feeding is affordable, feasible, acceptable, sustainable and safe (AFASS) ${ }^{6}$ National AIDS control organization recommends exclusive breastfeeding for first 6 months to all neonates of HIV 
positive mothers except for situations where breastfeeding cannot be done like maternal death or severe maternal illness. ${ }^{4}$

Postgraduate Institute of Medical Education and Research (PGIMER), Chandigarh is a referral center for HIV positive pregnant women in North India. Till 2013 (before the current NACO 2013 guidelines were adopted), if pregnant women were eligible for antiretroviral therapy (ART) for their own health, they were provided with the triple drug regimen (zidovudine or AZT + lamivudine or $3 T C+N V P)$. If ineligible, they were offered an alternate ARV prophylaxis regimen to reduce MTCT. We prospectively followed up all HIV positive pregnant women who delivered from June 2010 to December 2011 in order to observe maternal and neonatal outcome, including perinatal transmission rate.

\section{MATERIALS AND METHODS}

This prospective study was conducted in the Department of Obstetrics and Gynecology, PGIMER, Chandigarh. Women visiting the Antenatal Clinic were counseled and screened for HIV infection by ELISA tests at the ICTC. Of these, the HIV positive women who delivered in PGIMER from June 2010 to December 2011 were recruited for this study. Depending on the clinical assessment of HIV disease and CD4 count, they were offered either ARV prophylaxis (group 1) or ART (group 2). The criteria for ARV prophylaxis was CD4 $>350$ cells $/ \mathrm{mm}^{3}$ and WHO clinical stage 1 or 2 . Antiretroviral therapy was given to women with CD 4350 cells $/ \mathrm{mm}^{3}$ or WHO clinical stage 3 or 4 .

Group 1 women received ARV prophylaxis according to WHO 2006 regimen which included tablet AZT $300 \mathrm{mg}$ twice a day from 28 weeks period of gestation (POG) and a combination of AZT $600 \mathrm{mg}+3 \mathrm{TC} 150 \mathrm{mg}+\mathrm{NVP}$ $200 \mathrm{mg}$ at onset of labor or 3 hours prior to CS. A 7 days 'tail' of AZT $300 \mathrm{mg}$ + 3TC $150 \mathrm{mg}$ twice a day was continued for 1 week postpartum to the mother. Group 2 women were put on triple drug regimen throughout pregnancy and continued lifelong (tablet AZT $300 \mathrm{mg}$ $\mathrm{BD}+3 \mathrm{TC} 150 \mathrm{mg}$ BD + NVP $200 \mathrm{mg}$ BD; NVP was given OD for initial 2 weeks). Those with CD4 counts $<200$ cells $/ \mathrm{mm}^{3}$ were given prophylaxis against opportunistic infections (Pneumocystis carinii pneumonia) with $160 \mathrm{mg}$ trimethoprim and $800 \mathrm{mg}$ sulfamethoxazole once daily. All neonates received a single dose of syrup NVP $(2 \mathrm{mg} / \mathrm{kg})$ within 72 hours of birth plus syrup AZT $4 \mathrm{mg} / \mathrm{kg}$ 12 hourly for 6 weeks.

All women were followed regularly in the antenatal clinic. At 32 to 34 weeks POG, the risks and benefits of vaginal delivery $v$ s elective CS (to reduce perinatal HIV transmission) were discussed. In addition, exclusive breastfeeding $v s$ exclusive replacement feeding (with AFASS criteria) was explained so that these women could make an informed choice regarding these options. All women received a course of broad spectrum antibiotics during labor and delivery for 1 week. Delivery details, neonatal outcome and complications during the postpartum period were noted. The mothers and neonates were followed for 6 months. The neonates received routine immunization, PCP prophylaxis with co-trimoxazole (from 6 weeks to 18 months) and were tested for HIV by DNA PCR at 4 to 6 weeks of age. The blood sample was obtained by heel-prick and dried blood spot prepared. These samples were processed at the NACO accredited laboratory at AIIMS, New Delhi. If DNA PCR was negative, it was repeated at 4 to 6 months of age. If DNA PCR was positive, it was repeated immediately for confirmation of HIV positive status. All HIV positive babies were registered at the pediatric ART center and treated with ART. HIV negative babies were advised to undergo HIV ELISA at 18 months of age as a confirmatory test.

Descriptive statistics was used to define baseline variables. To compare the proportion of neonates with HIV infection born to mothers on ARV vs mothers on HAART, Chi-square test or Fisher's-Exact test was used. Among mothers on ARV and HAART, Chi-square test or Fisher's-Exact test were used to compare the proportion of neonates with HIV infection, who are born through vaginal delivery vs CS. The statistical analysis was carried out using Statistical Package for Social Sciences (SPSS Inc., Chicago, IL, version 17.0 for Windows. All statistical tests were two-sided and were performed at a significance level of $\mathrm{p}<0.05$.

\section{RESULTS}

A total of 5355 antenatal women were screened for HIV antibodies from June 2010 to December 2011; out of these 66 were found to be HIV positive. Thirty-five women who delivered in PGIMER were recruited for this study after obtaining a written consent (Flow Chart 1). In addition, of the women who reported directly in labor in the emergency area of PGIMER, 15 were found to be HIV positive (direct delivery group). Thus, a total of $81 / 5355$ women were HIV positive, making the antenatal HIV seroprevalence rate to be $1.5 \%$.

Table 1 shows the demographic data. Overall, 65.7\% women were between the age group of 26 to 30 years. There was total 10/35 (28.5\%) serodiscordant couples in the present study, and each group had a similar proportion of serodiscordant couples. In group 1, among the six serodiscordant couples, four women had been married a second time after the death of the first husband due to AIDS. Hence, they had probably acquired infection 


\begin{tabular}{|c|c|c|c|c|}
\hline & & Group 1 (ARV) $n=21(\%)$ & Group $2(A R T) n=14(\%)$ & $p$-value \\
\hline \multirow[t]{2}{*}{ Mean age (years) } & Woman & 27.05 & 26.86 & 0.84 \\
\hline & Husband & 31.3 & 30.07 & 0.137 \\
\hline \multicolumn{5}{|l|}{ Educational status } \\
\hline \multirow[t]{3}{*}{ subject } & Illiterate & $6(28.5)$ & $2(14.2)$ & \\
\hline & Up to secondary level & $9(42.7)$ & $9(64)$ & \\
\hline & Senior secondary or more & $6(28.5)$ & $3(21.3)$ & \\
\hline \multirow[t]{3}{*}{ Husband } & Illiterate & $1(4.7)$ & $2(14.2)$ & \\
\hline & Up to secondary level & $15(71.6)$ & $6(42.6)$ & \\
\hline & Senior secondary or more & $5(23.7)$ & $6(42.6)$ & \\
\hline \multirow[t]{3}{*}{ HIV status of husband } & Positive & $14(66.6)$ & $10(71.4)$ & 0.814 \\
\hline & Negative & $6(28.5)$ & $4(28.6)$ & 0.708 \\
\hline & Not known & $1(4.8)$ & 0 & \\
\hline Likely risk factor for HIV & Heterosexual route & $18(85.7)$ & $12(85.7)$ & 0.22 \\
\hline \multirow[t]{3}{*}{ infection } & Not known & 3 & & \\
\hline & Obstetric intervention & & 1 & \\
\hline & Occupational exposure & & 1 & \\
\hline \multirow[t]{2}{*}{ Occupation (women) } & Housewife & 20 & 13 & 1 \\
\hline & Working & 1 & 1 & 1 \\
\hline \multirow[t]{4}{*}{ Occupation (men) } & Laborer & 12 & 5 & 0.214 \\
\hline & Farmer & 1 & 4 & 0.133 \\
\hline & Driver & 2 & 0 & 0.505 \\
\hline & Shopkeeper & 6 & 5 & 0.721 \\
\hline
\end{tabular}

Flow Chart 1: Depicting recruitment of HIV positive pregnant women in the present study

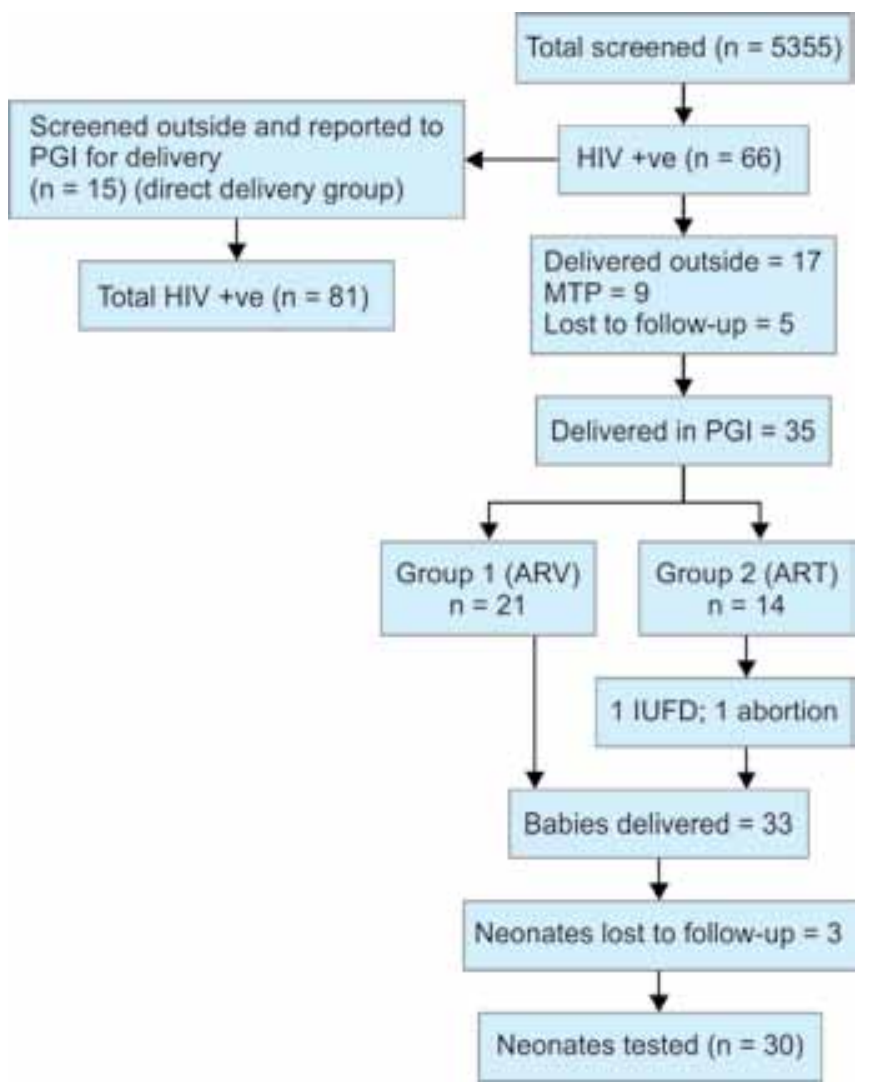

by heterosexual transmission from their first husband. In the remaining two, the cause of acquisition of HIV infection could not be ascertained. Of the four serodiscordant couples in group 2, the probable cause of HIV acquisition was heterosexual transmission in two women. In the other two, the probable source was exposure during a prior emergency CS, dilatation and curettage and mucosal exposure to an HIV positive patient's blood. Thus, $30 / 35(85.7 \%)$ women had acquired HIV infection by heterosexual route in the two groups.

Table 2 depicts the laboratory investigations of the study patients at recruitment. In group 1, 15/21(71.4\%) women had their baseline CD4 count between 351 to 500 cells $/ \mathrm{mm}^{3}$ and $6 / 21(28.6 \%)$ had a baseline CD4 count of $>500$ cells $/ \mathrm{mm}^{3}$. In group $2,11 / 14(78.5 \%)$ women had a baseline CD 4 count of $\leq 350$ cells $/ \mathrm{mm}^{3}$. Three women of group 2 had a CD4 count of $>350$ cells $/ \mathrm{mm}^{3}$ at their first visit during pregnancy. Of these 3 , two women had already been receiving ART for 3 years prior to conception which was continued during the pregnancy. The mean hemoglobin of women in group 1 was $10.4 \pm 1.02 \mathrm{gm} / \mathrm{dl}$ which was similar to the mean hemoglobin of women in group $2(9.98 \pm 1.11 \mathrm{gm} / \mathrm{dl})$. The mean total leukocyte count, platelet count, liver function tests, renal function tests and other routine investigations were also normal in all women except for one woman in group 2 who was found to be VDRL positive and received three doses of benzathine penicillin at weekly interval as treatment for the same.

Table 3 depicts the obstetric details of the patients of the two groups. In group 2, one woman had spontaneous abortion at 16 weeks period of gestation and one had an intrauterine fetal demise at 33 weeks POG. Thus, MTCT 
Table 2: Investigations of the HIV positive pregnant women

\begin{tabular}{lllll}
\hline & & Group 1 (ARV) $n=21(\%)$ & Group 2 (ART) $n=14(\%)$ & $p$-value \\
\hline CD4 count (cells $/ \mathrm{mm}^{3}$ ) & $<250$ & 0 & $4(28.5)$ & $7(50)$ \\
& $251-350$ & 0 & $2(14.2)$ \\
& $351-500$ & $15(71.4)$ & $1(7.1)$ & $298(202-334)$ \\
& $>500$ & $6(28.5)$ & $9.98 \pm 1.11$ & 0.201 \\
Hemoglobin $(\mathrm{gm} / \mathrm{dl})$ & Median and (IQR) & $470(384-533)$ & $7522 \pm 1524$ & 0.226 \\
Total leukocyte count $\left(\mathrm{cells} / \mathrm{mm}^{3}\right)$ & Mean \pm SD & $10.4 \pm 1.02$ & 0.95 \\
Platelet count $\left(\right.$ lakh $\left./ \mathrm{mm}^{3}\right)$ & Mean \pm SD & $6940 \pm 1251$ & $2.05 \pm 0.6186$ & $0.07 \pm 0.5288$ \\
\hline
\end{tabular}

Table 3: Obstetric details of the HIV positive pregnant women

\begin{tabular}{lll}
\hline Mode of delivery & $\begin{array}{l}\text { Group 1 (ARV) } \\
n=21\end{array}$ & $\begin{array}{l}\text { Group 2 (ART) } \\
n=12\end{array}$ \\
\hline Elective CS & $14 / 21(66.6 \%)$ & $9 / 12(75 \%)$ \\
Emergency CS & $1 / 21(4.8 \%)$ & $1 / 12(8.4 \%)$ \\
Vaginal & $6 / 12(28.5 \%)$ & $2 / 12(16.6 \%)$ \\
DROM range & 30 minutes 3 hours & 1 and 24 hours \\
DROM mean & $62.85 \pm 53.45$ & $\begin{array}{l}\text { Not calculated in } \\
\text { view of wide range }\end{array}$ \\
Mean DOL & 8.2 hours \pm 2.39 & 5 hours \\
Mean birth weight & $2.729 \pm 0.488$ & $2.825 \pm 0.55$ \\
\pm SD & & $38 \pm 0.66$ \\
$\begin{array}{l}\text { POG at delivery } \\
\text { (weeks) Mean } \pm \text { SD }\end{array}$ & $38.4 \pm 0.785$ & \\
\hline
\end{tabular}

could be seen in $12 / 14$ women. A total of $23 / 33(69.6 \%)$ opted for elective CS as the mode of delivery and the remaining $10(30.4 \%)$ chose to deliver vaginally out of whom two required emergency CS for fetal bradycardia. The duration of rupture of membranes (DROM) was $<2$ hours in these two women. Total 8 women delivered vaginally, 6 belonging to groups 1 and 2 women of group 2. One baby in group 1 was HIV positive among the women who delivered vaginally and the median duration of DROM was 30 minutes compared to HIV negative 5/ 6 (50 minutes), but the difference was statistically not significant $(\mathrm{p}=0.351)$. The difference in the duration of labor was also not significant $(\mathrm{p}=0.632)$. In group 2, both vaginally delivered babies were HIV negative. The mean POG at delivery and the mean birth weight were similar in the two groups. All the women in group 1 received AZT $600+3$ TC150 + sdNVP 200 mg 3 hours before CS or at onset of labor and in group 2 continued their prescribed ART regimen. All women in the two groups received broad-spectrum antibiotics in the intrapartum and postpartum period. All women in the study chose to give replacement feeding to their newborns after being counseled regarding the benefits and risks of replacement feeds. All babies of these women received sd-NVP syrup ( $2 \mathrm{mg} / \mathrm{kg}$ wt.) within 72 hours of birth and syrup AZT $4 \mathrm{mg} / \mathrm{kg}$, 12 hourly started within 6 to 12 hours after delivery and continued till 6 weeks. All infants received co-trimoxazole prophylaxis started from 4 to 6 weeks of age until HIV infection was excluded.

Human immunodeficiency virus testing for performed in 30 babies as 3 women refused testing for their babies. Table 4 shows the neonatal outcomes. A total of 4/30 babies were found to be HIV positive on DNA PCR test, making the overall perinatal transmission rate of $13.3 \%$. Similar proportion of women had delivered by elective CS in the two groups (group 1, 13/20 or $65 \%$ and group $2,7 / 10$ or $70 \% ; p=1)$. Overall, 20 women delivered by elective CS and 8 delivered vaginally in the two groups. Among the elective CS, $2 / 20(10 \%)$ and among the vaginal deliveries, $1 / 8(12.5 \%)$ babies were HIV positive. This difference was statistically not significant $(p=1)$. In group 1 , among the women who underwent an elective CS, the perinatal transmission was $7.7 \%$ compared to $16.7 \%$ in women who delivered vaginally. In group 2 , both vaginally delivered babies were HIV negative, hence such rates could not be assessed. One HIV positive baby in group 1 was delivered by an emergency CS.

Table 4: Distribution of HIV DNA PCR results of the babies in the two groups

\begin{tabular}{|c|c|c|c|c|c|c|c|c|c|}
\hline \multirow{2}{*}{$\frac{D N A \text { PCR of babies }}{\text { Positive }}$} & \multicolumn{4}{|l|}{$\begin{array}{l}\text { Group } 1 \\
(A R V) n=20\end{array}$} & \multicolumn{4}{|c|}{$\begin{array}{l}\text { Group } 2 \\
(A R T) n=10\end{array}$} & \multirow{2}{*}{$\frac{p \text {-value }}{1}$} \\
\hline & $3(15 \%)$ & & & & $1(10 \%)$ & & & & \\
\hline \multirow[t]{2}{*}{ Negative } & $17(85 \%)$ & & & & $9(90 \%)$ & & & & \\
\hline & Elective CS & \multicolumn{2}{|c|}{ Vaginal delivery } & Em CS & Elective CS & \multicolumn{2}{|c|}{ Vaginal delivery } & Em CS & \\
\hline Positive & $1(7.7 \%)$ & \multicolumn{2}{|c|}{$1(16.7 \%)$} & 1 & $1(14.3 \%)$ & \multicolumn{2}{|l|}{0} & \multicolumn{2}{|l|}{0} \\
\hline \multirow[t]{3}{*}{ Negative } & $12(92.3 \%)$ & \multicolumn{2}{|c|}{$5(83.3 \%)$} & 0 & $6(85.7 \%)$ & \multicolumn{2}{|c|}{$2(100 \%)$} & 1 & \\
\hline & & Med & Med & & & Med & Med & & \\
\hline & & DOL & DROM & & & DOL & DROM & & \\
\hline Positive & & 6 hours & 30 minutes & & & - & - & & \\
\hline Negative & & 8 hours & 50 minutes & & & 5 hours & 1 and 2 & & \\
\hline
\end{tabular}


Regarding the maternal outcome, two women in group 2 required blood transfusion, one immediately post LSCS due to increased operative blood loss and the other 15 days post CS due to secondary PPH. One woman in group 1 expired 40 hours after elective CS at 39 weeks. She was in WHO class I with CD4 count of 661, and was on AZT prophylaxis from 28 weeks. Preoperatively, ARV prophylaxis and intravenous broad spectrum antibiotics were given. She was transfused one unit of platelet concentrate as her platelet count was $78000 / \mathrm{mm}^{3}$. Her CS was uneventful and postoperatively period smooth till 16 hours postoperatively with adequate urine output. At 30 hours postoperatively, she was found to have anuria for last 14 hours and her condition deteriorated (gradually worsening hypotension, tachycardia, tachypnea and pyrexia). She was put on ventilatory support, however she died 10 hours later. After a detailed discussion, the cause of death was assigned as acute fulminant sepsis, possibly due to platelet transfusion. No other woman in any of the two groups had wound infection or pyrexia or any other morbidity.

\section{DISCUSSION}

The present observational study was conducted in HIV positive pregnant women who received either ARV to prevent perinatal transmission of HIV or ART for their own HIV disease. The antenatal HIV seroprevalence rate was found to be $1.5 \%$ which is slightly higher than $1 \%$ as reported by the UNAIDS Epidemiology fact sheet 2004 and NACO 2005. ${ }^{1,7}$ This may be explained by PGIMER, Chandigarh being a referral institute in North India.

The mean age of the women in our study was 26.97 years, which was similar to mean age of 351 Argentinean HIV infected women reported by Duran et al (27 years), ${ }^{8}$ of 418 Ethiopian women reported by Zinash et al ( 27 years $)^{9}$ and of $5882 \mathrm{HIV}$ positive women from UK and Ireland reported by Townsend et al (29.8 years). ${ }^{10}$ Nearly onefourth of women in the present study were illiterate as compared to 45 to $50 \%$ illiteracy rates in studies from African women. ${ }^{9}$ Majority were homemakers. Husbands of more than two-third of the women were drivers, farmers or laborers and had studied up to class 10th or less. More than $85 \%$ of the study patients had probably acquired HIV infection via the sexual route which is similar to that reported by Adrinas et al among Argentinean women. ${ }^{8}$ These women may have been at a higher risk for HIV acquisition due to promiscuous sexual behavior of their male partners.

In group 1, the median CD4 count was $477 / \mathrm{mm}^{3}$ in women whose babies were HIV negative and $386 / \mathrm{mm}^{3}$ in women whose babies were HIV positive but difference was not statistically significant $(\mathrm{p}=0.22)$. In group 2 , only one baby was HIV positive and CD4 count of the mother was $352 / \mathrm{mm}^{3}$ compared to $297 / \mathrm{mm}^{3}$ in women whose babies were HIV negative. Garcia-Tejedor et $\mathrm{al}^{11}$ reported that CD4 count $<500 / \mathrm{mm}^{3}$ increased the HIV transmission three times when the mother did not take ART. However, no difference was observed if mother were receiving HAART. A similar trend was observed in group 1 (ARV prophylaxis) of the present study in which higher HIV transmission rate was observed with lower $\mathrm{CD} 4$ count. Overall, $62 \%$ of the study patients were anemic, which is much more than prevalence of anemia among HIV uninfected women. ${ }^{12}$ As expected, in group $2,78.5 \%$ women had anemia which may be explained by their advanced HIV disease state.

In the present study, elective CS reduced MTCT from 16.7 to $7.7 \%$ in ARV prophylaxis group. In group $2 \mathrm{ART}$, no difference was noted between the outcomes in elective CS vs vaginal delivery. This result is in agreement with data from the European surveillance study by Townsend et al who showed that there was no significant effect of route of delivery on the perinatal transmission among women on combination ART. ${ }^{10}$ Townsend et al observed the HIV transmission rate to be only $0.8 \%$ among women on combination ART at least for 14 days. Overall 5 out of 33 or $15 \%$ neonates weighed less than $2.5 \mathrm{Kg}$ (low birth weight) in the present study which was similar to previous rate of $14.6 \%$ reported by Cooper et al. ${ }^{13}$

The ARV protocol offered at our institute has been followed since 2006 when WHO reported this to be most efficacious among the abbreviated regimens suggested for developing countries with MTCT rate of $4 \%$ or less reported by Dao et al. ${ }^{14}$ World Health Organization guidelines 2006 recommended that women who are not eligible for triple drug ART for their own disease should receive a combination prophylaxis antiretroviral regimen, preferably AZT from 28 weeks of gestation; AZT, 3TC and a single dose NVP during delivery; and AZT and 3TC for 7 days (tail) after delivery to reduce the development of NVP resistance. Newborn infants should receive a single dose of NVP and 1 to 4 weeks of AZT depending on the duration of treatment received by the mother. In the present study, all women in group $1(n=21)$ received this WHO 2006 ARV protocol, including the tail.

The overall perinatal transmission rate of $13.3 \%$ (15\% in group 1 and $10 \%$ in group 2) at 6 months of age in the present study is not low, considering the high rate of elective CS (70\%) and replacement feeding (100\%) among these women. In the ACTG 076 trial in USA, Connor et al reported MTCT at 18 months to be $8.3 \%$ in women receiving AZT from 14 weeks, intrapartum intravenous AZT and AZT to infant for 6 weeks. ${ }^{2}$ Lallement et al reported 
MTCT as $4.1 \%$ in women who received AZT from 28 weeks, intrapartum AZT + single dose NVP and AZT + single dose NVP to infant for 6 weeks. ${ }^{15}$ We could not find a reason for a higher rate of MTCT in the present study except that differences exist among different population groups. Direct comparison of MTCT rates among different trials is difficult because the patient population is from different geographical areas. However, a trend toward a lower rate in group 2 who are rather at a higher risk of MTCT reflects the likely benefit of maternal ART combination regimens given over a longer duration antenatally over abbreviated regimens of AZT monotherapy with addition of sd-NVP at labor onset. This observation is mentioned by the WHO 2010 guidelines also.

Based on the new recommendations of WHO (June 2013), National AIDS Control Program as a policy adopted the more efficacious multi-drug ART for PPTCT. ${ }^{4}$ These updated National Guidelines for PPTCT (NACO 2013) recommend to initiate lifelong triple drug ART regimen for all pregnant and breastfeeding women living with HIV, regardless of CD4 count or WHO clinical stage, both for their own health and to prevent vertical transmission, with additional HIV prevention benefits. This would also help in maximum coverage for those needing treatment for their own health, avoid stopping and starting drugs with repeat pregnancies, provide early protection against mother-to-child transmission in future pregnancies, reduce the risk of HIV transmission to serodiscordant couples and improve maternal health. These new guidelines are an endeavor of the Government of India to work toward the achievement of the global target of 'Elimination of the new HIV infection among children' by 2015.

\section{REFERENCES}

1. National Guidelines for HIV care and treatment in children. NACO 2005.

2. Connor EM, Sperling RS, Gelber R, Kiselev P, Scott G, O'Sullivan MJ, VanDyke R, Bey M, Shearer W, Jacobson RL. Reduction of maternal-infant transmission of human immunodeficiency virus type 1 with zidovudine treatment: Pediatric AIDS Clinical Trials Group Protocol 076 study group. Engl J Med 1994;331:1173-1180.

3. World Health Organization. Antiretroviral therapy of HIV infection in infants and children in resource-limited settings: towards universal access. Recommendations for a public health approach. Geneva: World Health Organization; 2006.

4. Updated guidelines for Prevention of Parent to Child Transmission (PPTCT) of HIV using Multi Drug Anti-retroviral Regimen in India. NACO, December, 2013.

5. American College of Obstetricians and Gynecologists. ACOG committee opinion scheduled Cesarean delivery and the prevention of vertical transmission of HIV infection. Int J Gynaecol Obstet 2001;73:279-281.

6. World Health Organization. WHO HIV and infant feeding technical consultation consensus statement. Geneva: WHO; 2006.

7. UNICEF India-The children-HIV/AIDS. Available at: www. unicef.org/India/children_2358.htm.

8. Duran AS, Ivalo SA, Hakim A, Masciottra FM, Zlatkes R, Adissi L, Neatoni JD, Marcelo H Losso. Prevention of mother to child HIV transmission. Med 2006;66(1):24-30.

9. Zinash Moges, Alemayehu A. Factor associated with readliness to VCT utilization among pregnant women attending antenatal clinics in northwestern Ethiopia: a health belief model approach. Thiop J Health Sci 2011 Aug;21(Suppl 1): 107-115.

10. Townsend CL, Cortina-Borja M, Peckham CS. Low rates of mother-to-child transmission of HIV following effective pregnancy interventions in the United Kingdom and Ireland, 2000-2006. AIDS 2008 May;22(8):973-981.

11. Garcia-Tejedor A, Maiques V, Perales A, Lopez-Aldeguer J. Influence of highly active antiretroviral treatment (HAART) on risk factors for vertical HIV transmission. Acta Obstet Gynaecol Scand 2009;88(8):882-887.

12. Samuel TM, Thomas T, Finkelstein J, Bosch R, Rajendran R, Virtanen SM, Srinivasan K, Kurpad AV, Duggan C. Correlates of anaemia in pregnant urban South Indian women: a possible role of dietary intake of nutrients that inhibit iron absorption. Public Health Nutr 2013 Feb;16(2):316-324.

13. Cooper ER, Charurat M, Mofensen L, et al. Combination antiretroviral strategies for the treatment of pregnant HIV 1 infected women and prevention of perinatal of perinatal HIV 1 transmission. J Acquire Immune Defic Syndr 2002 Apr;29(5):484-494.

14. Dao H, Mofensen LM, Ekpini R, Gilks CF, Barnhart M, Bolu O, Shaffer N. International recommendations on antiretroviral drugs for treatment of HIV infected women and prevention of mother-to-child HIV transmission in resource limited settings: 2006. Am J Obstet Gynecol 2007 Sep;197(Suppl 3):542-555.

15. Lallemant M, Jourdain G, Le Coeur S, Kim S, Koetsawang S, Comeau AM, Phoolcharoen W, Essex M, McIntosh K, Vithayasai V. A trial of shortened zidovudine regimens to prevent mother to child transmission of human immunodeficiency virus type 1: Perinatal HIV Prevention Trial (Thailand) investigators. N Engl J Med 2000 Oct 5;343(14):982-991. 